\title{
Ciencia y tecnología españolas en Internet: valoración a través de la presencia de organismos públicos españoles y de sus revistas electrónicas
}

\author{
Victor Manuel Pareja, Ana González, Isidro Aguillo
}

Arbor CLXII, 639 (Marzo 1999), 367-390 pp.

Se presenta un estudio sobre la presencia de los organismos de Investigación y Desarro. llo adscritos al sistema público de financiación, así como de las sociedades científicas. Se analiza además la situación de revistas electrónicas visibles en Internet editadas por estas instituciones. En ambos casos se ofrece también una valoración geográfica por Comunidades Autónomas, y en el caso de las revistas electrónicas una evaluación temática. Se suministra un directorio con los títulos y direcciones en Internet de las revistas electrónicas localizadas.

\section{Introducción}

Internet y muy especialmente el correo electrónico y el World Wide Web han revolucionado la forma en que los investigadores acceden y comunican la información y los resultados de su actividad. Ello ha abierto las puertas a nuevas posibilidades que tendrán su aplicación en muchos campos, dando lugar a nuevos métodos digitales de difusión de información científica y académica (boletines y revistas electrónicas); de almacenamiento y preservación de la misma (bibliotecas virtuales) y, en fin, a la irrupción de la Sociedad de la Información (tele-enseñanza, teletrabajo o telemedicina). 
Esta revolución va acompañada de una insólita explosión del volumen de información disponible (http://www.neci.nj.nec.com/homepages/lawrence/websize.html) y en circulación (http://www.cybergeography.org/atlas/geographic.html). En la actualidad, según datos propios, la Internet de los contenidos ha alcanzado los 400 millones de páginas Web (verano de 1998) y se duplica en un período de alrededor de 7-8 meses.

También, en lo tocante a la Internet física y de acuerdo con los datos suministrados por Network Wizards (http://www.nw.com) en su último Internet Domain Survey, correspondiente a julio de 1998, el número de ordenadores conectados a Internet ascendía a más de 36 millones en todo el mundo. Por lo que se refiere a España, en este mismo concepto ya disponía de 300.000 máquinas a primeros de diciembre de $1998{ }^{1}$, ocupando el lugar decimosexto con respecto a otros países y aglutinando el $0,7 \%$ mundial. (Tabla I).

TABLA I. Distribución de páginas web por regiones

\begin{tabular}{|c|c|c|}
\hline \multirow{2}{*}{ INTERNACIONALES } & \multicolumn{2}{|l|}{47,8} \\
\hline & Dominio com & 36.1 \\
\hline NORTE AMERICA & 15,4 & \\
\hline & $\begin{array}{l}\text { Estados Unidos (1) } \\
\text { Canadá (5) }\end{array}$ & $\begin{array}{l}13,1 \\
2,2 \\
\end{array}$ \\
\hline UNIÓN EUROPEA & 17,7 & \\
\hline & $\begin{array}{l}\text { Alemania (3) } \\
\text { Reino Unido (4) } \\
\text { España (16) } \\
\end{array}$ & $\begin{array}{l}4,2 \\
3,0 \\
0,7 \\
\end{array}$ \\
\hline OTROS EUROPA & 4,5 & \\
\hline & $\begin{array}{l}\text { Suiza (12) } \\
\text { Noruega (18) }\end{array}$ & $\begin{array}{l}0,9 \\
0,7 \\
\end{array}$ \\
\hline IBEROAMÉRICA & 1,6 & \\
\hline & Brasil (13) & 0,9 \\
\hline RESTO MUNDO & 13,0 & \\
\hline & $\begin{array}{l}\text { Japón (2) } \\
\text { Australia (7) } \\
\text { Corea (11) }\end{array}$ & $\begin{array}{l}7,7 \\
1,7 \\
1,0\end{array}$ \\
\hline
\end{tabular}

Porcentajes estimados sobre el total de los primeros 60 países $(+95 \%$ del total) calculado desde el motor Altavista (19 nov 98). Entre paréntesis la posición del país. Los dominios internacionales (com, net, org e int) se podrían asignar en un gran porcentaje también a Estados Unidos. 
Ciencia y Tecnología españolas en Internet...

Por otra parte, el número global de usuarios de Internet, según estimaciones de NUA Internet Survey (http://www.nua.ie), correspondería a 151 millones (diciembre 1998), o lo que es lo mismo el 3,7\% de la población mundial. En nuestro país, de acuerdo con el Estudio General de Medios que midió la audiencia en la Red en el período abril/mayo de 1998, disponemos de algo más de 2.200 .000 usuarios que tienen acceso ${ }^{2}$.

Aunque este acceso a Internet ha mejorado sustancialmente, las previsiones de crecimiento obligan a la adopción de técnicas más potentes para su tratamiento. Esto es especialmente importante en el ámbito de la comunicación científica donde la no duplicación de esfuerzos y el adecuado establecimiento de prioridades tienen importantes consecuencias para su financiación.

Los investigadores interesados en la evaluación de los resultados de la I+D y los gestores de las políticas científicas son cada vez más conscientes del importante peso específico que está adquiriendo Internet para su labor. En el pasado, los métodos cuantitativos desarrollados por la bibliometría se demostraron lo suficientemente sólidos para que su aplicación a la descripción y navegación por Internet ofrezca ahora grandes posibilidades. Este es el campo donde actúa la cibermetría, el estudio cuantitativo de los procesos de comunicación en Internet.

El método más somero de análisis cibermétrico se basa simplemente en cuantificar la presencia en la Red, y en concreto en la World Wide Web, de las instituciones relacionadas con actividades científico-técnicas. En esto precisamente consiste la primera parte de este trabajo: evaluar el estado de la ciencia española en Internet a partir de una visión panorámica de la presencia de instituciones públicas dedicadas al sector. Para profundizar un poco más en esta evaluación y como segunda parte de este análisis, se resalta el estudio de las revistas científicas en formato electrónico, cuya edición depende o bien de un organismo público o bien de sociedades científicas, habida cuenta de la estrecha relación que éstas poseen con el ámbito universitario.

\section{Presencia en Internet de organismos públicos del sistema español de ciencia y tecnología}

Si bien a finales de 1996 las universidades, encabezadas por la Politécnica de Valencia, copaban los primeros lugares en cuanto a la concentración del número de ordenadores en la Red, dos años después hay que mirar al cuarto lugar para encontrarse con aquélla, advirtiéndose ya la llegada de las potentes operadoras privadas de telefonía 
y los proveedores a esos primeros puestos, como es el caso de Retevisión, que concentra, con el $5,4 \%$, el mayor número del total de máquinas.

Esto no quiere decir que el mundo universitario-científico se haya quedado estancado en cuanto a su crecimiento en infraestructura en Internet, sino que se ha seguido produciendo uniformemente esa tendencia ascendente. Así por ejemplo, la antes citada UPV ha llegado a superar durante este período de dos años en más del doble la cantidad de ordenadores de que disponía: de 4.355 a 9.592 . Y valga también decir que el CSIC, a pesar de que ha descendido su posición del séptimo al undécimo puesto, sigue manteniendo un $2 \%$ del número de ordenadores.

La metodología a seguir para cuantificar la presencia de organismos públicos de $\mathrm{I}+\mathrm{D}$ no se fundamenta sin embargo en los datos de la Internet física o de infraestructuras, sino más bien en una Internet lógica, que se focalice en los contenidos. De esta forma, lo que se pretende es localizar sedes web, es decir, «aquel conjunto de páginas ligadas jerárquicamente, que constituyen una unidad diferenciada e independiente, tanto en función de contenidos (unidad documental) como de organización responsable (unidad institucional)", según hemos definido ya en algún trabajo anterior ${ }^{3}$

De manera que la captación de sedes web o unidades documentales objeto del análisis no puede realizarse de forma exhaustiva si no es únicamente a través de la propia navegación, ya que muchas de ellas cuelgan jerárquicamente de servidores institucionales de mayor rango. Simultánea a la navegación se realiza la indización del recurso localizado gracias a la opción de marcadores de que dispone Netscape Navigator, que permite el almacenamiento automático de direcciones y su posterior tratamiento documental mediante la asignación de identificadores en un campo de descripción ilimitado. Asimismo se procede a la normalización del título del recurso, que también se registra automáticamente coincidiendo con el título que le dio el creador de la página HTML, y que en la gran mayoría de los casos no sirve de cara a una indización pertinente.

Las instituciones que se examinan para valorar su presencia en Internet son las siguientes:

1. Universidades, incluyendo también las privadas por razones de homogeneidad, y divididas en facultades, escuelas técnicas, departamentos y grupos de investigación, así como aquellas otras instituciones que dependen orgánicamente de ellas, tales como 
Ciencia y Tecnología españolas en Internet...

institutos universitarios, oficinas de transferencia de investigación, bibliotecas, oficinas de programas europeos, centros mixtos y fundaciones.

2. Organismos Públicos de Investigación, ya sean de carácter estatal, autonómico o local, asimismo subdivididos en OTRIs y bibliotecas.

3. Consejo Superior de Investigaciones Cientificas, el OPI más importante de nuestro país que, por razones del volumen de su presupuesto y dimensión se trata aparte. Se consideran sus delegaciones, sus centros e institutos, sus centros mixtos, sus departamentos y grupos de investigación y sus bibliotecas.

\section{Sociedades Científicas}

Otra perspectiva de análisis es la clasificación geográfica por Comunidades Autónomas, de forma que se puede estimar la distribución de la presencia de sedes de acuerdo con su localización.

\section{Resultados}

En primer lugar, es necesario comentar que los datos extraídos para esta cuantificación corresponden a enero de 1998 y que en algún momento se puede establecer una comparación con aquéllos que arrojaban estudios anteriores ${ }^{4}$.

Tomando en consideración la figura 1 se puede apreciar que, de todos los organismos dedicados a investigación con financiación pública, la Universidad es con mucho la que mayor cantidad de sedes web ofrece, un $84 \%$, marcando un gran desequilibrio con respecto al CSIC (9\%), a las Sociedades Científicas (4\%) y a los OPI (3\%).

Por separado, la evolución experimentada en un año por el ámbito universitario se computa como muy positiva, pues se eleva a un $78 \%$ más de sedes presentes en la Red, a contar desde finales de 1996 hasta enero de 1998. Pero más grata es todavía la aportación del CSIC en esa misma línea ascendente, que aumenta en un $138 \%$, o la de las Sociedades Científicas, que logran duplicar su presencia. Menor cuantía consiguen los OPI, con solo un $34 \%$ de incremento. (Véase Tabla II). Con todo, en términos generales, se puede comprobar la tendencia ascendente de presencia en el web de toda la $\mathrm{I}+\mathrm{D}$ pública hasta en un $81 \%$. 
FIgURA 1. Presencia en Internet de Organismos de $\mathrm{I}+\mathrm{D}$

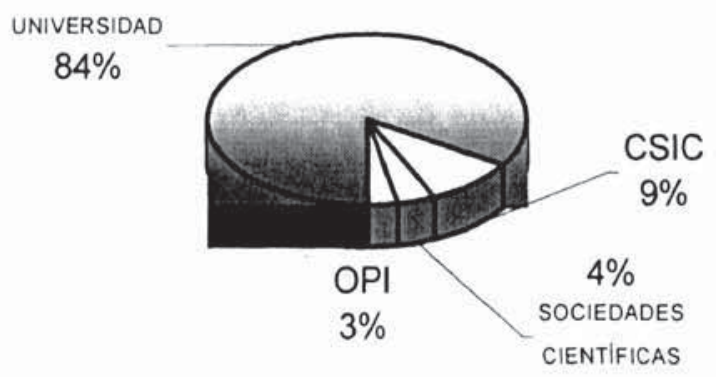

TABLA II. Incremento de sedes WEB

\begin{tabular}{|l|c|c|c|}
\hline & 1996 & 1998 & $\%$ \\
\hline Universidad & 970 & 1.732 & 78 \\
\hline CSIP & 78 & 186 & 138 \\
\hline OPI & 44 & 59 & 34 \\
\hline Sociedades científicas & 42 & 84 & 100 \\
\hline Total & 1.136 & 2.061 & $81 \%$ \\
\hline
\end{tabular}

\subsection{Universidades}

Si bien en 1996 todavía algunas universidades no habían iniciado la carrera por estar en Internet, actualmente ya todas estas instituciones académicas poseen un servidor central, incluso las privadas y las de más reciente creación, concienciadas desde su nacimiento de la necesidad pujante que es hallarse presente en la Red. El número de todas ellas asciende en la actualidad a 63 .

Para analizar las universidades se han disgregado en entidades que dependen de ellas orgánica y estructuralmente o de alguna otra manera. Por supuesto no se han reflejado todas las sedes, sino aquéllas que se hallen vinculadas con las actividades de investigación-docencia. La inclusión de las bibliotecas o centros de documentación está motivada por el papel que aportan de apoyo a la investigación. Idénticas razones valen para las Fundaciones universitarias, ya sean generales o de empresa-universidad, que tienden a dedicarse a la estimulación de actividades científicas. Los centros mixtos son una categoría que obedece simplemente a su pertenencia a dos o más instituciones.

En la tabla III se presentan todas las subdivisiones consideradas y los datos, en términos absolutos, correspondientes al número de sedes encontradas en los dos períodos temporales ya aludidos. Se ha optado 
por separar Facultades de Escuelas debido a la distinta naturaleza y diferentes campos del conocimiento que casi siempre contemplan cada una de ellas. Por otra parte, se han cuantificado conjuntamente departamentos, grupos de investigación, áreas, secciones, unidades, etc. bien dentro de Facultades, bien dentro de Escuelas, por razón de sus características estructurales similares.

TABLA III. División institucional Universidad

\begin{tabular}{|lccc|}
\hline & 1996 & 1998 & $\%$ Aum. \\
\hline Universidades & 50 & 63 & 26 \\
Facultades y Colegios adscritos & 82 & 136 & 65 \\
Escuelas Técnicas y Universitarias & 71 & 123 & 73 \\
Departamentos, grupos de investigación, áreas, laboratorios de & 448 & 723 & 61 \\
Facultades & & & \\
Departamentos, grupos de investigación, áreas, laboratorios de & 172 & 372 & 116 \\
Esc. Téc. y Univ. & 13 & 41 & 215 \\
Centros mixtos & 71 & 115 & 61 \\
Institutos universitarios y Centros de investigación & 13 & 30 & 130 \\
Otros & 35 & 97 & 177 \\
Bibliotecas universitarias & 10 & 13 & 30 \\
Oficinas programas europeos & 5 & 19 & 28 \\
Fundaciones & 970 & 1.732 & 78 \\
\hline TOTAL & & & \\
\hline
\end{tabular}

De la observación de la tabla se deduce que las entidades con mayor aumento han resultado ser las Fundaciones y los centros mixtos, superando el $200 \%$. También digna de destacar es la mayor presencia de las bibliotecas y centros de documentación en el seno de la Universidad (177\%). Los departamentos y grupos de investigación de las Escuelas han experimentado una subida de un $116 \%$ en contraste con los pertenecientes a Facultades, pese a que esta categoría sigue contemplando el mayor número de sedes web en valores absolutos.

\subsection{CSIC}

El CSIC es un Organismo Público de Investigación multidisciplinar que desarrolla su actividad científica a través de 109 centros e institutos repartidos por toda España, de los cuales, 26 poseen la condición de centros mixtos con universidades o Comunidades Autónomas. 
Para el análisis del CSIC, se cuenta con unos datos más actuales todavía que corresponden a junio de 1998. De los Centros-Institutos que ostentan la naturaleza de centros propios del CSIC ya se encuentran en Internet un $77 \%$, y de los mixtos un $73 \%$. Además, se han sumado al servidor de la oficina central las delegaciones situadas en Aragón y Andalucía, aunque todavía falta la mayor parte de éstas. En la tabla IV se muestran los datos correspondientes.

TABLA IV. Presencia del CSIC en Internet

\begin{tabular}{|lcc|}
\hline & $\mathrm{N}^{\circ}$ sedes & $\%$ \\
\hline Central y Delegaciones & 3 & $23 \%$ \\
Centros e Institutos & 64 & $77 \%$ \\
Centros mixtos & 19 & $73 \%$ \\
Departamentos y grupos & 51 & $8 \%$ \\
Departamentos y grupos Centros mixtos & 15 & $17 \%$ \\
Bibliotecas & 19 & $20 \%$ \\
Unidades de apoyo a la investigación & 15 & $9 \%$ \\
\hline Totales & 186 & $18 \%$ \\
\hline
\end{tabular}

En el estudio de 1996, la incidencia de penetración del CSIC se computaba en solamente un tercio de los centros e institutos (36), habiendo disfrutado, eso sí, de un notable ascenso a lo largo de ese año, pues para marzo sólo tenía registrado en la Red un 10\% (9).

Lo que resulta todavía bastante pobre es la no incorporación de muchos departamentos y grupos de investigación con sedes web propias - solamente el $8 \%$ de ellos las poseen-, y máxime si los comparamos con la ingente cantidad de sus homónimos universitarios. Sin embargo, la participación de bibliotecas del CSIC ha subido a un $20 \%$ con relación a 1996, donde sólo estaban presentes un $4 \%$ de las mismas.

\subsection{Organismos Públicos de Investigación}

Aunque según la Ley de la Ciencia de 1986, los OPI son por definición más o menos una docena de centros, hemos querido hacer extensible el concepto a aquellos organismos dedicados a la investigación in extenso sin diferenciar la administración que ostenta su titularidad. De esta manera los efectivos pertenecientes a esta categoría se amplían y además se conceden ciertos méritos a la administración autonómica, que posee el mayor número de entes con presencia en el web, o se hace palpable 
la gran carencia que en el ámbito local (ayuntamientos o diputaciones) existe todavía en este sentido.

Así, la tabla V nos muestra la cantidad de sedes en Internet que ostentan los OPI, habiendo diferenciado también las Oficinas de transferencia de investigación o las bibliotecas. Los organismos de naturaleza autonómica concentran un $54 \%$ de la presencia total de OPI en Internet. $\mathrm{Y}$ los estatales, un $34 \%$. Muy lejos está y queda mucho por hacer en la administración local para superar una única sede web.

TABLA V. Organismos Públicos de Investigación

\begin{tabular}{|lc|}
\hline & $N^{\circ}$ sedes \\
\hline Locales & 1 \\
Autonómicos & 32 \\
Estatales & 20 \\
Otris & 2 \\
Bibliotecas & 4 \\
\hline Bibliotecas & 59 \\
\hline
\end{tabular}

Con todo, comparando la presencia de los OPI con la del espectro universitario y del CSIC y haciendo todas las salvedades oportunas sobre la base de la proporcionalidad entre los tres ámbitos, la diferencia es clara y habla negativamente de los primeros aunque sólo nos remitiéramos a la variable del incremento ya vista en la tabla $2(138 \%$ CSIC, $78 \%$ Universidad, 34\% OPI).

\subsection{Sociedades Científicas}

De las Sociedades Científicas, muy vinculadas a los departamentos universitarios y de ahí la justificación para incluirlas en este estudio, se puede únicamente decir que en nuestro país disponen actualmente de sede web 84 de ellas, habiéndose duplicado su número desde 1996.

\subsection{Análisis geográfico}

En la figura 2, se puede apreciar la distribución geográfica de las sedes web en las doce primeras Comunidades Autónomas. En Madrid y en Cataluña se da la mayor concentración, con un 24,2 y un $17,8 \%$ respectivamente. Van seguidas de Andalucía que acumula el 14,8\% y ya un poco más lejos la Comunidad Valenciana con un $9,8 \%$. Por abajo, 
el dato más significativo es que en La Rioja sólo existía una sede, que representa sólo el $0,04 \%$ de organismos de $\mathrm{I}+\mathrm{D}$ en Internet.

Figura 2. Distribución geográfica de sedes

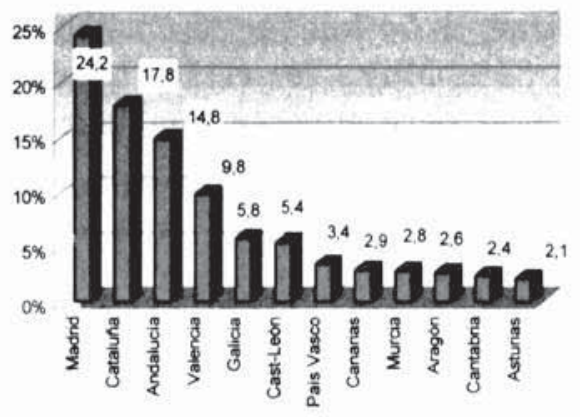

En el anexo I se presenta una tabla que detalla más pormenorizadamente todas las sedes web pertenecientes a cada Comunidad Autónoma, interrelacionadas también por instituciones. De esos datos se extrae como relevante el que Andalucía posea mayor número de bibliotecas universitarias ${ }^{20} \mathrm{y}$ de OTRIs ${ }^{7}$ que Madrid o Cataluna. Asimismo, que el $70 \%$ de los webs de Baleares pertenezcan a Departamentos y Grupos de Investigación de Escuelas Técnicas o Universitarias, siendo una de las seis Comunidades, junto con Canarias, Cantabria, Asturias, Navarra y Castilla-La Mancha, en las que se invierte la relación de mayor número de departamentos de facultades sobre los de escuelas.

En Madrid se halla una amplia representación de los organismos del CSIC, lo que propicia por consiguiente una mayor presencia en el web alcanzando un $47 \%$ de sedes encontradas; lo mismo que ocurre para Sociedades Científicas, que concentran un $41 \%$.

Contrastando los datos de evaluación geográfica con los disponibles para 1996 (según la tabla VI), podemos comentar el ascenso de Madrid - del 20 al 24,2\%- y de Castilla-León - del 3,6 al 5,4\%-. Así como el descenso de Cataluña -1,4 puntos - Andalucía, Valencia y Canarias - 1,6 puntos-

TABLA VI. Crecimiento según comunidades autonómas

\begin{tabular}{|l|r|r|}
\hline \multicolumn{1}{|c|}{ C.C.A.A. } & 1996 & \multicolumn{2}{|c|}{1998} \\
\hline Madrid & $20 \%$ & $24,2 \%$ \\
\hline Cataluña & $19,2 \%$ & $17,8 \%$ \\
\hline Andalucia & $15,5 \%$ & $14,8 \%$ \\
C. Valenciana & $11 \%$ & $9,8 \%$ \\
\hline Castilla-León & $3,6 \%$ & $5,4 \%$ \\
\hline Canarias & $4,5 \%$ & $2,9 \%$ \\
\hline
\end{tabular}


Ciencia y Tecnología españolas en Internet...

\section{Las revistas electrónicas editadas por los organismos de I+D}

Las nuevas tecnologías y en concreto Internet están transformando las formas y procesos tradicionales de comunicación científica. El uso del correo electrónico, los foros de debate, la formación de comunidades virtuales, la interactividad, la inmediatez y el fácil acceso a la información que proporciona el entorno Web, la superación de limitaciones geográficas, son sólo unos apuntes momentáneos de lo que estamos por ver en años venideros. Pero sin duda, la revista electrónica como medio de difusión de los conocimientos científicos es el máximo exponente de este cambio.

Las revistas electrónicas son ya una realidad que va madurando a medida que pasa el tiempo. Cómo evolucionará el fenómeno y su generalización en coexistencia con el formato tradicional, dependerá del papel que quieran o tengan que asumir los diferentes agentes que intervienen en el proceso de comunicación, tal y como apunta Alice Keefer $(1997)^{5}$ :

- los científicos, remisos a enviar artículos a revistas que todavía no gocen de la aceptación suficiente;

- las editoriales, que habrán de resolver problemas de producción, márketing, cobro y derechos de autor;

- los usuarios lectores que demandan más facilidad en su utilización, $\mathrm{y}$

- las bibliotecas, con dudas sobre el almacenamiento de los artículos a largo plazo.

Entre las ventajas que las revistas electrónicas aportan a la comunicación científica se encuentran el ahorro en los costes, más facilidad para la edición, la incorporación de elementos multimedia, la eliminación de los canales de intermediación o distribución clásicos y una mayor interactividad entre el autor y sus colegas o lectores. Además, son un instrumento más ecológico, proveen más facilidad de acceso a la información por la inmediatez y por el uso del hipertexto en la estructura interna de los documentos. Las búsquedas pueden ser más ágiles y puede darse la consulta de números anteriores en forma de archivo permanente, así como la posibilidad de recibir la suscripción en el buzón de correo electrónico.

Asimismo, de acuerdo con $\operatorname{Ramos}^{6}$, a diferencia de la edición tradicional en que los costes de distribución determinan que el número de lectores disminuya con el aumento de la distancia, en la edición electrónica surge el concepto de una comunidad lectora con los mismos hábitos e intereses con independencia de su situación geográfica. 
Entre los inconvenientes, se encuentran los problemas que plantea la función de archivo histórico del conocimiento científico que propicia la edición tradicional y que con las revistas electrónicas surgen ciertas dudas sobre su conservación, dada la propia naturaleza mutable de la información en Internet. Otro problema de las revistas en formato digital es el derivado de la inconveniencia de la lectura; todavía se prefiere el papel como medio más amigable para leer. También la lentitud que se da en incluir las revistas electrónicas en los servicios tradicionales de índices y resúmenes o la rápida evolución de los estándares de edición y las nuevas herramientas de programación, favorecidos por el desarrollo tecnológico, pero que para las comunidades científicas suponen cierta inestabilidad. La normalización y la calidad científica son otros de los obstáculos para su generalización.

En este sentido, y para preservar el rigor de los artículos publicados en la Red, no hay motivo para que no se pueda mantener la figura de los comités de expertos que revisen los trabajos. Todo depende de la voluntad de los editores para evitar procesos endogámicos en la publicación.

Con todo, aún no existiendo estos filtros de calidad, el problema sería de fácil solución si se sigue, según preconizan ciertos autores como Harnad (1995) ${ }^{7}$, la filosofía de entender la publicación en Internet como un proceso de revisión más democratizador y abierto, en el que los propios lectores, con sus observaciones y críticas, serían los que en sí dictaminasen sobre la calidad del artículo, lo que a su vez propiciaría que el autor conociese la repercusión de su labor y se abriesen nuevas vías de comunicación e interactividad sin intermediación editorial. Como señala Romero, "las ideas inteligentes son tan inteligentes sobre la superficie del papel como en la pantalla de un ordenador ${ }^{8}$ ". Para este autor, el que no se dé el gran salto a la edición electrónica se debe a motivos sociológicos y prejuicios de los propios científicos a que se vea amenazada la estabilidad de sus relaciones de grupo y de sus procesos de trabajo.

Por otra parte, los aspectos económicos que marcan la edición tradicional y que afectan al mundo académico público se verían sumamente alterados de cuajar a plenitud la posibilidad de publicar en revistas electrónicas sin merma del prestigio o de la calidad. De esta transformación habla Luis Fernando Ramos ${ }^{9}$ que destaca cómo en estas circunstancias el papel de los editores comerciales no tendría ya razón de ser. En el ámbito universitario, y por extensión en el de la I+D pública, las publicaciones electrónicas pueden fundamentar su éxito debido a que son fáciles y baratas de hacer, a la rapidez de su dis- 
tribución, a la facilidad de contabilidad de accesos (y por tanto, de valorar su impacto).

Hasta ahora, en el esquema convencional para la transmisión de la información científica, cuestión que también abordan Barrueco y García Testal ${ }^{10}$, ésta es proporcionada gratuitamente a las editoras privadas para que a su vez la pongan a disposición del mercado, de donde tienen que obtenerlas, previo pago, las propias instituciones en donde se genera esa información. De manera que la Universidad, por ejemplo, gasta cuantiosas sumas en suscripciones a revistas basadas en información que ella misma ha producido, con resultados de investigación que ella misma ha subvencionado, y que ha cedido gratis. Además, en este proceso «el autor - afirman García Testal y Barruecoentrega los derechos de copia de sus trabajos al editor, quien a cambio los difunde y obtiene un beneficio económico. De esta forma los derechos de copia sirven para proteger los intereses económicos de los editores, y el autor no tiene más remedio que aceptarlos si quiere ver sus trabajos publicados en las revistas de prestigio".

Con la edición electrónica todo este entramado comienza a tambalearse. Si un departamento, un profesor o un investigador edita sus propios trabajos directamente en las páginas web de su centro, la función del editor privado tiende a desaparecer en este entorno, al no tener control sobre la producción de la información, además de ganar rapidez, sencillez y ahorro y poder llegar a una audiencia potencial mundial.

Por todo lo dicho, el sector público debería estar muy interesado en la publicación por medios digitales para dar conocimiento de los resultados de su actividad investigadora en beneficio de toda la sociedad. Financiación pública, luego fines públicos. En este marco es dónde queremos insertar la justificación de la evaluación de revistas electrónicas en WWW, con el fin de comprobar el estado actual de la cuestión.

\subsection{Metodología}

Para los propósitos de este estudio se parte de un concepto de revista científica electrónica residente en un servidor informático y accesible por Internet, y que es publicada formal y estructuradamente bajo un título fijo con artículos de carácter periódico. Además, debe contar con una institución académico-científica como responsable de la edición que avale su rigor y calidad, o bien, en el caso de editores personales, que trabajen en ese tipo de organismos. 
Con el objeto de garantizar esta calidad, ya se ha comentado el papel que de hecho cumplen los comités científicos que se ocupan de la revisión formal e intelectual de los trabajos enviados a la revista.

Dentro de las revistas electrónicas se puede establecer una tipología:

Atendiendo al formato:

a) Solo existen en versión electrónica.

b) Existiendo en formato papel, disponen de una versión electrónica paralela.

Atendiendo al contenido:

a) Disponibles a texto completo.

b) Contiene solo resúmenes.

c) Solo sumarios.

Estas dos últimas presuponen una edición completa en papel o son de acceso restringido.

Atendiendo a la accesibilidad:

a) Gratuita y de acceso libre.

b) De acceso restringido a determinada comunidad.

c) Mediante pago.

Como en la primera parte del trabajo, la captura de recursos en formato web se hizo mediante la navegación por departamentos y centros académicos y de investigación. El tratamiento en el caso de las revistas electrónicas no se ha supeditado al concepto de sede, sino que se han almacenado y tratado simplemente con el requisito de que hubiese algunas páginas web informativas sobre la existencia de una revista científica y que suministrasen datos sobre su contenido.

El estudio no tiene como propósito hacer un análisis de la calidad del contenido de las revistas. Solamente se computan algunos indicadores que sirvan de muestra para determinar el estado de las revistas electrónicas. Estos indicadores son:

- el origen institucional,

- la procedencia geográfica,

- la temática,

- el idioma,

- si hay comité editorial,

- si existe versión en papel,

- la disposición del contenido, sea en texto completo, con resumen o con sumario solamente, y

- la periodicidad. 


\section{Ciencia y Tecnología españolas en Internet...}

En algún caso extremo, no se suministraban los datos necesarios para completar estos valores, como en el de que la publicación sea de acceso restringido o que en cuanto a la periodicidad no se ofrezca ninguna información, por lo que se ha optado por consignarla como de carácter irregular.

Los datos obtenidos corresponden también a enero de 1998.

\subsection{Resultados}

Se han localizado 118 revistas electrónicas, entre las que se incluyen también las dedicadas a las Ciencias Sociales y Humanas, así como a la Medicina Clínica. Como sea que nuestro estudio se va a centrar en las revistas electrónicas de Ciencias Experimentales y Tecnología, la muestra que se va a tratar desciende a 31 . Al ámbito universitario pertenecen el $48,4 \%$, mientras que un $25,8 \%$ las editan sociedades científicas. El CSIC participa en la edición de 19,4\% de las mismas y en último término los OPI contribuyen con únicamente el 6,4\% (Figura 3). El dato de la universidad como editor, pero con un porcentaje muy distinto (76\%), es el aportado en un estudio anterior llevado a cabo desde nuestro departamento sobre la presencia de revistas electrónicas en Ciencias Sociales y Humanidades, aunque también incluía una panorámica internacional en comparación con las tendencias que se daban en nuestro país ". En dicho estudio se consideraban editores públicos y privados.

Desde el punto de vista geográfico, a tenor de lo visto en la tabla VII, se aprecia la misma tónica que se daba en la presencia de organismos: la Comunidad de Madrid consigue aglutinar más de la mitad de las publicaciones digitales con el $51,6 \%$, seguida de Cataluña y Andalucía que disponen del mismo porcentaje, el $13 \%$. Como polo opuesto

FIGURA 3. Instituciones editoras de revistas científicas electrónicas

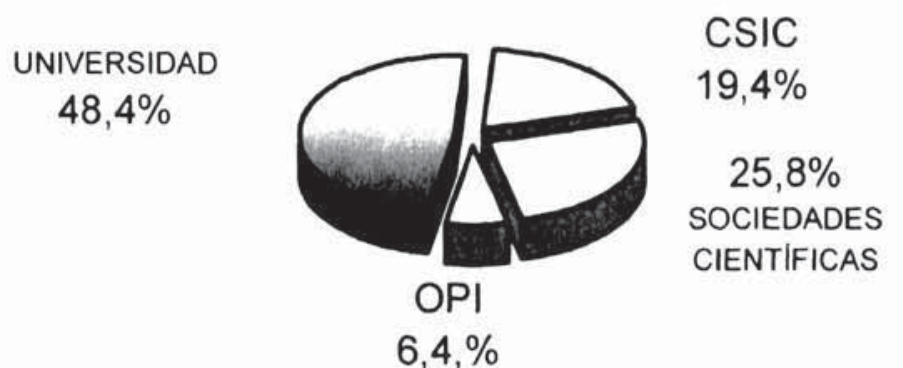


hay que significar que no fue encontrada ninguna publicación en nueve Comunidades Autónomas.

TABLA VII. Distribución por Comunidades Autónomas

\begin{tabular}{|l|c|c|}
\hline \multicolumn{1}{|c|}{ C.C.A.A. } & R.E & $\%$ \\
\hline Madrid & 16 & 51,6 \\
\hline Cataluña & 4 & 13,0 \\
\hline Andalucia & 4 & 13,0 \\
\hline Valencia & 2 & 6,4 \\
\hline País Vasco & 2 & 6,4 \\
\hline Asturias & 1 & 3,2 \\
\hline Castilla-León & 1 & 3,2 \\
\hline Navarra & 1 & 3,2 \\
\hline TOTAL & 31 & 100,0 \\
\hline
\end{tabular}

Por lo que respecta a la temática, han sido encontradas 11 materias distintas. Las Ciencias de la Vida condensan la mayor densidad con un $25 \%$. A continuación se sitúan las Matemáticas con un $12,5 \%$ de títulos y después Física, Química, Ciencias de la Tierra y Alimentación con un 9,3\% respectivamente. Otras disciplinas como Estadística, Agricultura e Informática poseen el $6,4 \%$.

$\mathrm{Si}$ tenemos en cuenta la perspectiva del idioma, encontramos (Figura 4) que las revistas monolingües constituyen el $68 \%$ del total en la siguiente proporción:

$\begin{array}{lll}\text { - monolingüe: } & 76 \% & \text { castellano } \\ & 24 \% & \text { inglés }\end{array}$

FIGURA 4.

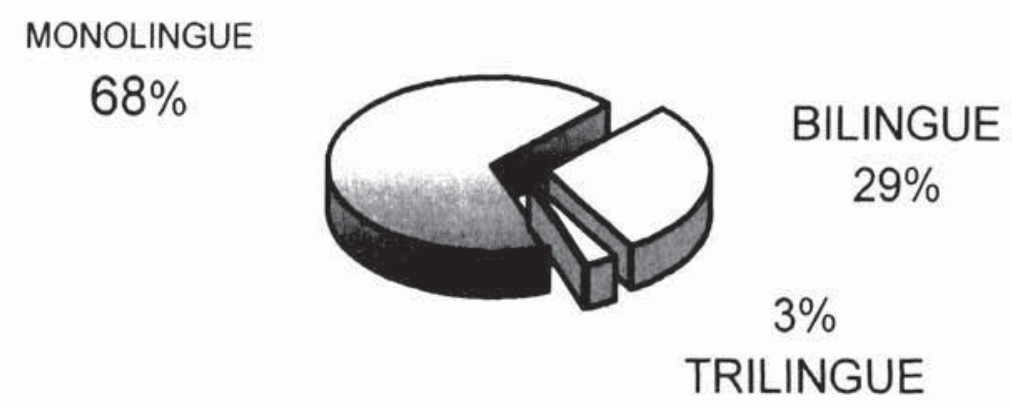


Ciencia y Tecnología españolas en Internet...

Las revistas bilingües halladas ascienden al $29 \%$ y han sido sólo correspondientes a castellano/inglés. El $3 \%$ es trilingüe y sólo en castellano/inglés/ catalán.

El $68 \%$ de las publicaciones estudiadas disponen de algún tipo de comité editorial, mientras que el $32 \%$ o bien no tiene o bien no viene expresado en la información general sobre la revista, lo que supone que alrededor de un tercio no suministra un aval para la calidad intelectual de los contenidos.

Cuentan con una versión en papel casi el $100 \%$ de la muestra; tan sólo una revista existía exclusivamente en formato electrónico. Y relativo al tipo de publicación que se observa (Figura 5), el 39\% suministraba el contenido a texto completo, el $29 \%$ proporcionaba resúmenes y el $23 \%$ solo sumarios. Un $9 \%$ no tenía disponible esta información o porque estaba en construcción, o porque eran revistan de acceso restringido y no se podía acceder sino era con contraseña por suscripción. Por último, queda significar la periodicidad, de la que se ha hallado casi toda la gama de casos posibles. El máximo lo copa la periodicidad irregular $(29 \%)$, bien porque sea así o porque no se haya podido determinar otra por falta de información. La periodicidad semestral es la segunda más frecuente agrupando el $16,2 \%$ de los títulos, seguida de la anual y de la trimestral con el $13 \%$ respectivamente. Mensuales, bimensuales y cuatrimestrales completan el montante de revistas analizadas (9,6\% cada una).

Figura 5. Tipo de contenido en las revistas científicas electrónicas

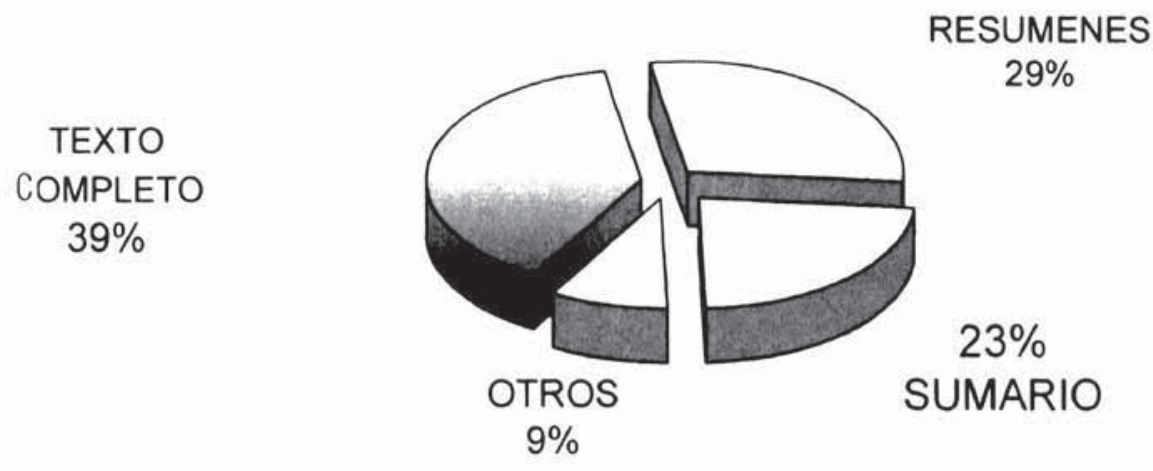




\section{CONCLUSIONES}

El sector público de $\mathrm{I}+\mathrm{D}$ español sigue presentando una tendencia de ascenso en términos de penetración en Internet, lo que significa que el mundo de la ciencia ve la Red con buenos ojos para, al menos, incluirse en ella. Está todavía por ver cómo se transformarán los procesos de transmisión de la información científica aprovechándola como medio de comunicación y en concreto a través de las revistas electrónicas.

El mundo de la Universidad es el que cuenta con mayor presencia y en constante aumento, aunque el CSIC es el organismo que más esfuerzo ha hecho en incrementar su presencia durante los últimos dos años, en detrimento de otros OPI, que siguen incorporando sedes web a bastante menor ritmo.

Las sesenta y tres universidades, tanto públicas como privadas, que se han contabilizado como existentes en nuestro país tienen ya un servidor central con presencia en Internet. Continúan siendo los departamentos y grupos de investigación quienes más sensibilizados están para mostrarse visibles y poner en funcionamiento sedes web. Los centros mixtos y las fundaciones son, sin embargo, los entes que han experimentado un mayor aumento, aunque su presencia en términos absolutos es todavía pobre. Pero particularmente importante es el ascenso de las bibliotecas universitarias o centros de documentación, de los que ya hay disponibles un centenar.

Más de dos tercios de los centros e institutos del CSIC se hallan incorporados en la WWW. Parece importante la concienciación que se viene dando en este sentido. Sin embargo, esta potenciación también debe extenderse a los departamentos, tomando como parangón el espectro universitario, que han sido los entes dentro del organigrama del CSIC que menos han emergido en cuanto a crecimiento $\mathrm{y}$ visibilidad independientes.

Las sociedades científicas han experimentado un efecto de duplicación en el período transcurrido desde diciembre de 1996 a enero de 1998.

Geográficamente, Madrid sigue a la cabeza de sedes web de $\mathrm{I}+\mathrm{D}$ público e incrementa su distancia con relación a Cataluña y Andalucía, que ocupan el segundo y el tercer lugar.

En otro orden de cosas, Internet está transformando los modos tradicionales de comunicación científica. La función de los editores comerciales particulares se puede ver muy trastocada con la consolidación de las revistas electrónicas como medio de difusión del conocimiento científico. Accesibles de forma gratuita para una au- 
Ciencia y Tecnología españolas en Internet...

diencia mundial y editadas fácilmente por parte de los propios departamentos y centros académico-científicos, pueden ser aprovechadas por las consabidas ventajas de interactividad y abaratamiento de costes y revolucionar así el mundo editorial en este sector.

De momento, y mientras asistimos a su evolución, el análisis realizado revela que la Universidad puede beneficiarse de este aprovechamiento, al ser el agente más comprometido en la edición de revistas electrónicas. El CSIC se verá obligado a dar un impulso tocante a las publicaciones digitales similar al manifestado para sus centros e institutos, con objeto de no seguir viéndose superado por las revistas de las sociedades científicas. Lo dicho también vale, y con más intensidad si cabe, para todos los demás OPI, habida cuenta de su poca participación como editores.

Geográficamente, se enumeran los mismos lugares y en el mismo orden que para los organismos de $\mathrm{I}+\mathrm{D}$, a saber Madrid, Cataluña y Andalucía, aunque con mayor diferencia en los valores acumulados en la primera de ellas, cuadruplicando a la segunda y tercera. Por abajo, también es destacable el que sólo ocho Comunidades Autónomas no dispongan de publicaciones electrónicas.

Es necesario que disminuya el carácter de monolingüismo, compartiendo los idiomas del estado con el inglés como caso mínimo, para que la publicación electrónica tenga visibilidad desde cualquier parte del mundo y sea mayor el impacto de la información científica que se publique. La situación de trilingüismo sería entonces el ideal a seguir. Aquí entraría en juego un rasgo diferencial con las publicaciones en papel, en las que es inviable esta circunstancia por el encarecimiento de costes.

Desde el punto de vista del tipo de publicación, es muy significativa la poca presencia de revistas electrónicas a texto completo, fenómeno consustancial a la falta de revistas de ciencias puras y experimentales nacidas estrictamente para la edición en el web.

Por eso, confiamos que el panorama de las revistas electrónicas se enriquezca a medida que se incremente el todavía escaso número de ellas que exclusivamente están en formato electónico, con lo cual estaríamos ya en la posición de pensar que Internet se había convertido en una plataforma muy eficaz, única e inigualable para la difusión del conocimiento científico. 


\begin{tabular}{|c|c|c|c|c|c|c|c|c|c|c|c|c|c|c|c|c|c|c|}
\hline 30 & \begin{tabular}{l} 
הี \\
\multirow{a}{*}{}
\end{tabular} & $\begin{array}{l}10 \\
\infty \\
- \\
-\end{array}$ & $\begin{array}{l}0 \\
0 \\
=\end{array}$ & $\begin{array}{l}\infty \\
\infty \\
\sigma^{\infty}\end{array}$ & 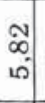 & 웜 & के & ज़ & $\begin{array}{l}\overline{0} \\
\text { N. }\end{array}$ & 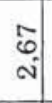 & 원 & & : & $\begin{array}{l}10 \\
-10 \\
-1\end{array}$ & & t) & $\stackrel{\Delta}{0}$ & 8 \\
\hline : & ஓ्ञ & \begin{tabular}{l}
0 \\
0 \\
\hdashline
\end{tabular} & ले & ลे & సิำ & $\exists$ & 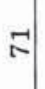 & ¿ & in & เด้ค & 워 & & ల్ల & స్ & $\stackrel{\infty}{\infty}$ & ה & - & $\begin{array}{l}\vec{\delta} \\
\text { ì }\end{array}$ \\
\hline 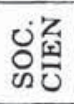 & 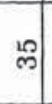 & $\stackrel{0}{2}$ & $\infty$ & 10) & -1 & m & -1 & $N$ & & $N$ & $\rightarrow$ & 0 & -1 & $\rightarrow$ & $\rightarrow$ & & & 苂 \\
\hline ڤ & $\stackrel{\infty}{\sim}$ & 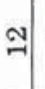 & $\nabla$ & is & -1 & & 0 & 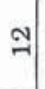 & & $\rightarrow$ & & & & & & & & 요 \\
\hline U్ & $\begin{array}{l}\infty \\
\infty\end{array}$ & ल्ల్| & ลิ & $\infty$ & ๓ & $\nabla$ & & $N$ & -1 & $\infty$ & $\nabla$ & ๓ & เด & & 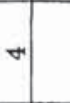 & $N$ & & 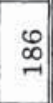 \\
\hline 斻 & $H$ & - & & $\nabla$ & & $N$ & & $\rightarrow$ & $\rightarrow$ & & & & & & & & & 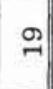 \\
\hline 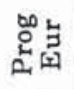 & $N$ & & $\rightarrow$ & $n$ & & & $\rightarrow$ & & & & & & & - & & & & $\stackrel{\mathscr{m}}{\sim}$ \\
\hline$\stackrel{\overrightarrow{0}}{ }$ & $\stackrel{20}{\sim}$ & $\stackrel{\infty}{\sim}$ & ㅇํ & 0 & -1 & $\bullet$ & 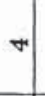 & N & $\infty$ & $\approx$ & $\sim$ & ก & & N & & & & ธิ \\
\hline$\vec{E}$ & $\nabla$ & 0 & - & $\nabla$ & m & $\rightarrow$ & & & $\rightarrow$ & & N & & & - & & & & ల్ల \\
\hline 菢宏 & $*$ & สี & in) & מ & $\rightarrow$ & N & & & & & -1 & & & & $\Rightarrow-$ & -1 & & $F$ \\
\hline 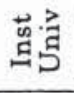 & $\vec{m}$ & ๙ & $\cong$ & \pm & 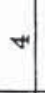 & 10 & क & H & -1 & $\rightarrow$ & N & N & -1 & $m$ & N - & & & $\stackrel{2}{=}$ \\
\hline 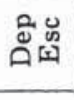 & ๕ & เฉ็ & กิ & 원 & $\stackrel{2}{\sim}$ & 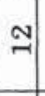 & 0 & ำ & 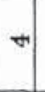 & $\infty$ & ๓ & $\stackrel{m}{\rightarrow}$ & -1 & $\approx$ & N & & & న్ల్ల \\
\hline 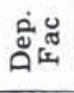 & $\neq$ & $\stackrel{\infty}{\sim}$ & 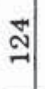 & 6 & 잔 & if & $\stackrel{\text { : }}{\sim}$ & $\infty$ & म্ল & $\Rightarrow$ & $\infty$ & o. & జ & $\nabla$ & ㅇ․ & & & $\underset{i}{\mathbb{N}}$ \\
\hline 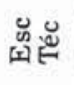 & 이 & จิ & $\div$ & $\stackrel{\infty}{\sim}$ & $\infty$ & 의 & $\infty$ & $\nabla$ & $\infty$ & $\infty$ & $\rightarrow$ & in & - & $\mathrm{N}$ & N 4 & & & 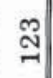 \\
\hline$\underset{\infty}{\infty}$ & เุ & 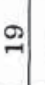 & ज゙ & $\exists$ & 0 & \pm & $\infty$ & $m$ & क) & $\infty$ & ๓ & $\infty$ & & + & $\infty-$ & -1 & & 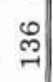 \\
\hline 5 & $\cong$ & 의 & 의 & D & $\infty$ & $\mathrm{N}$ & $\infty$ & $\sim$ & -1 & -1 & N & $\Rightarrow$ & - & $N$ & $\rightarrow-$ & -1 & & 8 \\
\hline 过 & $\begin{array}{l}\frac{\pi}{\pi} \\
\frac{\pi}{2}\end{array}$ & ' & 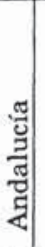 & : & 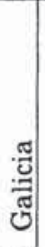 & 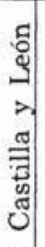 & 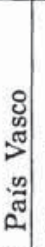 & 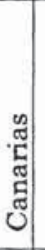 & 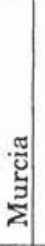 & 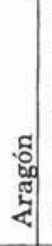 & 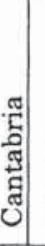 & 常 & ๓్ & : & 觖 & 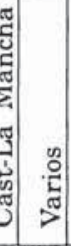 & $\mid \begin{array}{l}0 \\
0 \\
0 \\
0 \\
3 \\
3\end{array}$ & 胥 \\
\hline
\end{tabular}




\section{Ciencia y Tecnología españolas en Internet...}

ANExo II. Tabla de la temática de revistas electrónicas

\begin{tabular}{|l|c|}
\hline \multicolumn{1}{|c|}{ MATERIAS } & NoTIT. \\
\hline Ciencias de la Vida & 8 \\
\hline Matemáticas & 4 \\
\hline Física & 3 \\
\hline Química & 3 \\
\hline Ciencias de la Tierra & 3 \\
\hline Alimentación & 3 \\
\hline Estadística & 2 \\
\hline Agricultura & 2 \\
\hline Informática & 2 \\
\hline Arquitectura & 1 \\
\hline Ciencias Aeroespaciales & 1 \\
\hline TOTAL & $32 *$ \\
\hline
\end{tabular}

*Son 32 a pesar de que el número de revistas estudiadas sea 31 porque una revista da cobertura a dos temáticas distintas.

\section{Anexo III. Directorio de revistas electrónicas científicas}

\section{Agricultura}

TI: Boletin Informativo del INIA. “http://www.inia.es/boletines/boletines.htm".

ED: Instituto Nacional de Investigación y Tecnología Agraria y Alimentaria

TI: IRTA PORC's: Boletín Informativo del Sistema GTEP-IRTA.http://labor3.etsea.udl.es/IRTA/IRTAPORC/IRTAPORCAST.HTLM *

ED: Universitat de Lleida. ETS de Ingenieria. Departamento de Ingeniería Agroforestal.-IRTA

\section{Alimentación}

TI: Boletin Informativo del INIA. "http://www.inia.es/boletines/boletines.htm -

ED: Instituto Nacional de Investigación y Tecnología Agraria y Alimentaria

TI: Food Science and Technology International. Revista internacional de Ciencia y Tecnología de los Alimentos. "http://www.iata.csic.es/ -bibrem/revfsti.htm"

ED: CSIC. Instituto de Agroquímica y Tecnologia de los Alimentos

TI: Grasas y Aceites. Revista sobre grasas comestibles. "http://www.ig.csic.es/revis.htm"

ED: CSIC. Instituto de la Grasa

\section{Arquitectura}

TI: Cuadernos de Investigación Urbanistica. "http://www.aq. upm.es/uoVciu/ciu.html"

ED: Universidad Politécnica de Madrid. ETS Arquitectura. Departamento Urbanistica y Ordenación del Territorio.

\section{Ciencias Aeroespaciales}

TI: INTA.ES. Revista sobre el sector acroespacial. "http://www.inta.es/areas/inta.es/numero0/entrada htm. ED: Instituto Nacional de Técnica Aeroespacial 
Ciencias de la Tierra

TL Boletin de la Real Sociedad Española de Historia Natural. Sección Geologta. http://www.ucm.es/info/rsehn/pub/geo/pub-geo.htmn ED: Real Sociedad Española de Historia Natural

TI: Estudios Geologicos. Revista acerca de las Ciencias de la Tierra. «http://www.csic.es/estudios_geol/n

ED: CSIC. Museo Nacional de Ciencias Naturales

TI: Revista de Minas (RDM). Publicación sobre Laboreo, Metalurgia, Energía y Ciencias de la Tierra ahttp://www.etsimo.uniovi.es/rdm/w

ED: Universidad de Oviedo. Escuela Técnica Superior de Ingenieros de Minas

\section{Ciencias de la Vida}

TI: Antilia. Revista Española de Historia de las Ciencias de la Naturaleza y de la Tecnología. Revista de historia de la biología.

«http://www.ucm.es/info/antilia/m

ED: Universidad Complutense de Madrid. Facultad de Biología

T I: Boletín de la Real Sociedad Española de Historia Natural. Sección Biologia. whttp://www.ucm.es/info/rsehn/pub/bio/pub-bio.htm*

ED: Real Sociedad Española de Historia Natural

TI Ecosistemas. Revista de Ecologia y Medioambiente. "http://www.ucm.es/info/ecosistemas/"

ED: Universidad Complutense de Madrid. Facultad de Biología. Departamento Interuniversitario de Ecología

TI: Encuentros en la Biología. «http://www.uma.es/Estudios/Centros/Ciencias/publicaciones/encuentros/*

ED: Universidad de Málaga. Facultad de Ciencias

TI: Microbiologia SEM. «http://morgat.udg.es/microbsem/index.html»

ED: Sociedad Espanola de Microbiología

TI: Revista Española de Patologla. «http://www.unav.es/interpatpatolog/rev.htm"

ED: Sociedad Española de Anatomía Patológica; Sociedad Española de Citologia

TI: Scientia Marina. Revista Internacional de Ciencias Marinas. "http://www.icm.csic.es/scimar/sci_index.html» ED: CSIC. Instituto de Ciencias del Mar

TI: Zoea Newsletter. Revista de Desarrollo larvario de crustáceos. "http://sice.uca.es/icman/Zoeanewsletter.html ED: J.A. Cuesta y P.J. López González (Universidad de Sevilla. Facultad de Biológicas); J.I. González-Gordillo (Instituto de Ciencias Marinas de Andalucía).

\section{Estadística}

TI: Fuentes Estadisticas. Revista de Estadística.uhttp://www.festadisticas.fguam.es/"

ED: Universidad Autónoma de Madrid. Instituto Nacional de Estadística. Oficina Estadística de la Unión Europea

TI: Revista Método de Encuesta. Revista de Metodología de las Encuestas. "http://filabres.ualm.es/me/n

ED: Universidad de Almería

\section{Física}

TI: Anales de Ftsica. "http://www.ucm.es/info/rsef/texto/publicacion/anales/anales.html

ED: Real Sociedad Española de Física

TI: Revista Española de Fisica. "http://www.ucm.es/info/rsef/texto/publicacion/revista/revista_espanola.html"

ED: Real Sociedad Española de Física

TI: Revista Ingenieria del Agua. "http://www.upv.es/ria/index. de html"

ED: Universidad de Valencia. Departamento de Ingeniería Hidráulica y Medio Ambiente. Grupo Mecánica de Fluidos.

\section{Informática}

TI: Nueva Login. Revista de Informática. «http://www.gui.uva.es/login/»

ED: Universidad de Valladolid. Grupo Universitario de Informática 


\section{Ciencia y Tecnología españolas en Internet...}

TI: Sprinet. Revista de Informática. “http://gmx44.lg.ehu.es/documentos.htmV/diferente/rev.html" ED: Universidad del País Vasco. Servicio de Informática

\section{Matemáticas}

TI: Extracta Mathematicae. Revista de Matemáticas. «http://www.unex.es/extracta/extracta.html" ED: Universidad de Extremadura.Departamento de Matemáticas

TI: Publicacions Matematiques. "http://mat.uab.es/pubmat/welcome"

ED: Universidad Autónoma de Barcelona. Departamento de Matemáticas

TI: Revista Matemática. Revista de Matemáticas. "http://www.ucm.es/info/rmat"

ED: Universidad Complutense de Madrid. Facultad de Matemáticas

TI: Revista SIPROMA de Matemática Iberoamericana. "http://www.oei.es/mateiber.htm"

ED: Sociedad Iberoamerica para la Promoción de la Matemática

Química

TI: Afinidad. Revista de Química. "http://www.iqs.url.es/iqs/aiqs/afinid.htm"

ED: Instituto Químico de Sarria. Asociación de Químicos

TI: Anales de Quimica. ahttp://www.ucm.es/info/rsequim/anales.html"

ED: Real Sociedad Española de Química

TI: Revista de Plásticos Modernos. "http://www.revplasmod.com/"

ED: CSIC. Instituto de Ciencia y Tecnología de Polímeros

\section{Bibliografía y Notas}

1 Datos disponibles en ES-NIC (http://www.nic.es) Registro de nombres de dominio para su uso en el DNS de Internet.

2 Asociación para la Investigación de Medios de Comunicación. $2^{a}$ Ola de la En. cuesta General de Medios. "http://www.aimc.es/aimc/html/inter/3a.html». [Consulta: 3 diciembre 1998]

3 AGUILLO, I.F.: Hacia un concepto documental de sede web. IWE. El profesional de la información. (Barcelona) 1998, vol.7, n¹-2, p.p. 45-46.

4 PAREJA, V.M.; AGUillo, I.F.: Evolución de la presencia del sector público de I+D en Internet durante 1996. Libro de Ponencias del II Congreso Nacional de Usuarios de Internet e Infovía. 1997. Madrid. pp. 57-64.

5 KEEFER, Alice: La revista electrónica y su aceptación por parte del usuario final. En: Cid, P.; Baró, J. (eds.): Anuario Socadi de Documentación e Información 1997. Barcelona: Societat Catalana de Documentació i Informació, 1997, p. 185-190.

6 RAMOS, L.F. Las publicaciones electrónicas transformarán el sector de la edición científica y las funciones del bibliotecario en la Universidad. Cuadernos de Documentación Multimedia. 1997-1998, nº6-7. "http://www.ucm.es/info/multidoc/multidoc/revista/cuad6-7/ramos.htm".

7 HARNAD, S. Implementing peer review on the net: scientific quality control in scholarly electronic journals. En: Peek, R.; Newby, G. (eds.): Electronic Publishing confronts academia: the agenda for the year 2000. Cambridge MA: MIT Press, 1995.

8 Para comprobar por qué la comunidad científica desea tener control sobre sus procesos de comunicación y cómo esto sirve de obstáculo para el avance e implantación de las revistas electrónicas, se puede consultar el interesante artículo de Joaquín María Aguirre Romero "Las revistas digitales y la vida académica". Cuadernos de Documentación Multimedia. 1997-1998, n6-7. «http://www.ucm.es/info/multidoc/multi- 
doc/revista/cuad6-7/aguirre.htm". También examina los condicionantes e intereses que operan en los procesos de publicación dentro del ámbito científico-académico-editorial. Hace afirmaciones como que «el papel principal de las publicaciones en el escenario académico hace $[. .$.$] que estas se constituyan en una forma de control del flujo humanon$ 0 que «la comunidad académica es esencialmente conservadora. Sus relaciones internas, sus relaciones de grupo, giran sobre la publicación impresa. [...] ¿Está la comunidad académica preparada -mejor, dispuesta - para la entrada de nuevos canales de distribución que desestabilicen las relaciones internas? [...] La facilidad de publicación de las revistas electrónicas, la salida de grandes cantidades de material que aguardan en cajones a que alguien le dé el visto bueno para su publicación, supone un cambio drástico en las relaciones internas dentro del grupo académico-científico. Publicar, en la medida en que es una necesidad para todos, se convierte en una forma de poder. La forma más eficaz de no perder ese poder es despreciar o infravalorar los nuevos medios electrónicos".

9 RAMOS, L.F, op.cit.

10 GARCIA TESTAL, C.; BARRUECO, J.M: Algunos apuntes sobre edición científica electrónica y derechos de autor. IWE. El profesional de la información. (Barcelona) 1998, vol 7, n4, p.p. 7-10.

11 VILLALÓN, J.; AGUILLO, I.F.: Revistas electrónicas en Ciencias Sociales y Humanidades. Revista Española de Documentación Científica. (Madrid) 1998, vol 21, n³3, p.p. 303-316. 